PROCEEDINGS OF THE

AMERICAN MATHEMATICAL SOCIETY

Volume 126, Number 5, May 1998, Pages 1409-1413

S 0002-9939(98)04261-0

\title{
SPECTRAL AVERAGING AND THE KREIN SPECTRAL SHIFT
}

\author{
BARRY SIMON
}

(Communicated by Palle E. T. Jorgensen)

\begin{abstract}
We provide a new proof of a theorem of Birman and Solomyak that if $A(s)=A_{0}+s B$ with $B \geq 0$ trace class and $d \mu_{s}(\cdot)=\operatorname{Tr}\left(B^{1 / 2} E_{A(s)}(\cdot) B^{1 / 2}\right)$, then $\int_{0}^{1}\left[d \mu_{s}(\lambda)\right] d s=\xi(\lambda) d \lambda$, where $\xi$ is the Krein spectral shift from $A(0)$ to $A(1)$. Our main point is that this is a simple consequence of the formula $\frac{d}{d s} \operatorname{Tr}\left(f(A(s))=\operatorname{Tr}\left(B f^{\prime}(A(s))\right)\right.$.
\end{abstract}

Let $A$ and $C=A+B$ be bounded self-adjoint operators, and suppose that $B \geq 0$ and $B$ is trace class.. Then it is a fundamental result of Krein [5], [6], [7] that there is an $L^{1}$ function $\xi_{A, C}(\lambda)$ so that for any $C^{1}$ function $f$ (and, in particular, for all $\left.f \in C_{0}^{\infty}(\mathbb{R})\right)$,

$$
\operatorname{Tr}(f(C)-f(A))=\int f^{\prime}(\lambda) \xi_{A, C}(\lambda) d \lambda
$$

Here $\xi \geq 0$ and is uniquely determined by (1) and the condition that $\xi$ is $L^{1}$. This $\xi$ has compact support (if $A, C$ are bounded). Moreover, if $B$ is finite rank, then

$$
0 \leq \xi_{A, C}(\lambda) \leq \operatorname{rank}(B)
$$

and

$$
\lim _{s \rightarrow \infty}\left[\xi_{A, A+s B}(\lambda)-\xi_{A, A-s B}(\lambda)\right]=\operatorname{rank}(B) .
$$

In 1971, Javrjan [3] proved a remarkable formula for $\operatorname{rank}(B)=1$ : Let $B=$ $(\varphi, \cdot) \varphi$ and define $d \mu_{s}(\lambda)$ by $\int e^{i \lambda t} d \mu_{s}(\lambda)=\left(\varphi, e^{i t(A+s B)} \varphi\right)$ and

$$
d \eta(\lambda)=\int_{s_{0}}^{s_{1}}\left[d \mu_{s}(\cdot)\right] d s
$$

the average of the spectral measures for $A+s B$. Then, Javrjan's formula is

$$
d \eta(\lambda)=\xi_{A+s_{0} B, A+s_{1} B}(\lambda) d \lambda .
$$

In particular, by (3) we have

$$
\int_{-\infty}^{\infty}\left[d \mu_{s}(\lambda)\right] d s=d \lambda
$$

Javrjan only proved this for the special case of boundary condition variation of half-line Sturm-Liouville operators, but it easily extends to any rank one situation [9]. (5) in various guises has been repeatedly rediscovered by workers in the theory of

Received by the editors October 14, 1996.

1991 Mathematics Subject Classification. Primary 47B10, 47A60.

This material is based upon work supported by the National Science Foundation under Grant No. DMS-9401491. The government has certain rights in this material. 
random Hamiltonians; most notably, Wegner's estimates on the integrated density of states [10] and Kotani's formula on spectral averaging [4] are essentially (5).

Our main goal in this note is to provide a generalization of (4) to general positive trace class perturbations with a proof that is essentially trivial and provides a simple approach even for the rank one case. The extension is not new - it appears in a paper of Birman-Solomyak [1] which seems not to be widely known. I decided to write this note partly to advertise their result, but also because my proof is transparent while their proof relies on an elaborate mechanism of Stieltjes double operator integrals.

A key realization of our approach is that the spectral averaging is a consequence of the formula

$$
\frac{d}{d s} \operatorname{Tr}(f(A(s)))=\operatorname{Tr}\left(A^{\prime}(s) f^{\prime}(A(s))\right) .
$$

This trace class chain rule is formally evident, and is discussed, for example, in [2]. We will prove the version we need below.

For our most basic result, let $A(s)$ be a family of bounded self-adjoint operators for $s \in\left[s_{0}, s_{1}\right]$ so that:

(1) The weak derivative $B(s)=\frac{d A}{d s}$ exists.

(2) $B(s)$ is trace class and positive.

(3) $s \mapsto B(s)$ is continuous in trace norm.

Let $E_{s}(\Delta)$ be the spectral projections for $A(s)$ and define

$$
d \mu_{s}(\lambda)=\operatorname{Tr}\left(B(s)^{1 / 2} d E_{s}(\lambda) B(s)^{1 / 2}\right)
$$

and

$$
d \eta(\cdot)=\int_{s_{0}}^{s_{1}}\left[d \mu_{s}(\cdot)\right] d s
$$

Our main result is:

Theorem 1. Under the hypotheses (1)-(3), $A\left(s_{1}\right)-A\left(s_{0}\right)$ is trace class. Moreover,

$$
d \eta(\lambda)=\xi_{A\left(s_{0}\right), A\left(s_{1}\right)}(\lambda) d \lambda,
$$

where $\xi_{A\left(s_{0}\right), A\left(s_{1}\right)}$ is the Krein spectral shift.

Remarks. 1. If $\operatorname{Ran}(B(s))$ is cyclic for $A(s)$, then $d \mu_{s}(\lambda)$ is a complete spectral measure in that for any measurable $\Delta \subset \mathbb{R}, E_{s}(\Delta)=0$ if and only if $\mu_{s}(\Delta)=0$.

2. We will get (8) via (9) below and then appeal to (1). That the measure defined by (1) is absolutely continuous with respect to Lebesgue measure is Krein's deep result. We assume it here.

3. That $B(s) \geq 0$ isn't essential for the theorem to hold, since we can take $d \mu_{s}(\lambda)=\operatorname{Tr}\left(\operatorname{sgn}(B)|B|^{1 / 2} d E_{A(s)}(\lambda)|B|^{1 / 2}\right)$; but $d \mu_{s}(\lambda)$ is then not positive, and so control of $\int d \mu_{s}(\lambda) d s$ does not tell one much about the individual $d \mu_{s}(\lambda)$.

We will prove Theorem 1 from

Proposition 2. Under the hypotheses (1)-(3) for any $s, A(s)-A\left(s_{0}\right)$ is trace class, and for any $f \in C_{0}^{\infty}(\mathbb{R}), f(A(s))-f\left(A\left(s_{0}\right)\right)$ is trace class. Moreover,

$$
s \mapsto \operatorname{Tr}\left(f(A(s))-f\left(A\left(s_{0}\right)\right) \equiv g(s)\right.
$$

is $C^{1}$ and

$$
\frac{d g}{d s}=\operatorname{Tr}\left(B(s) f^{\prime}(A(s))\right)
$$


Proof of Theorem 1 given Proposition 2. The definition of $d \mu_{s}(\lambda)$ and the spectral theorem imply that for any $s$ and $h \in C_{0}^{\infty}(\mathbb{R})$,

$$
\operatorname{Tr}(B(s) h(A(s)))=\int h(\lambda) d \mu_{s}(\lambda) .
$$

Thus,

$$
\begin{aligned}
\int f^{\prime}(\lambda) d \eta(\lambda) & =\int_{s_{0}}^{s_{1}} d s \int f^{\prime}(\lambda) d \mu_{s}(\lambda) \\
& =\int_{s_{0}}^{s_{1}} \frac{d g}{d s} d s \quad \text { by }(9) \\
& =g\left(s_{1}\right) \\
& =\operatorname{Tr}\left(f\left(A\left(s_{1}\right)\right)-f\left(A\left(s_{0}\right)\right)\right) \\
& =\int f^{\prime}(\lambda) \xi_{A\left(s_{0}\right), A\left(s_{1}\right)}(\lambda) d \lambda .
\end{aligned}
$$

Since $f$ is an arbitrary $C_{0}^{\infty}$ function, we conclude that, for a constant $c$,

$$
d \eta(\lambda)=(\xi(\lambda)+c) d \lambda
$$

But $d \eta$ and $\xi$ have compact support, so $c=0$.

Proof of Proposition 2. By the weak differentiability,

$$
A(s)-A(t)=\int_{t}^{s} B(u) d u
$$

where the function is a weak integral (i.e., (11) holds if matrix elements are taken inside the integral). But by the hypothesis on $B$, the trace norm Riemann integral exists, and so (11) must hold in that sense. This implies that $A(s)-A(t)$ is trace class and

$$
\frac{A(s+\delta)-A(s)}{\delta} \rightarrow B(s)
$$

in trace norm.

Fix $u \in \mathbb{R}$. Then DuHamel's formula

$$
e^{i u A(s)}-e^{i u A(t)}=i u \int_{0}^{1} e^{i \theta A(t)}(A(s)-A(t)) e^{i(1-\theta) A(s)} d \theta
$$

holds initially weakly, but as above in trace norm sense. It follows that

$$
\frac{d\left(e^{i u A(s)}-e^{i u A\left(s_{0}\right)}\right)}{d s}=i u \int_{0}^{1} e^{i \theta A(s)} B(s) e^{i(1-\theta) A(s)} d \theta
$$

in trace class sense. Thus,

$$
\frac{d}{d s} \operatorname{Tr}\left(e^{i u A(s)}-e^{i u A\left(s_{0}\right)}\right)=i u \operatorname{Tr}\left(e^{i u A(s)} B(s)\right),
$$

and this implies

$$
\operatorname{Tr}\left(e^{i u A(s)}-e^{i u A\left(s_{0}\right)}\right)=i u \int_{s_{0}}^{s} \operatorname{Tr}\left(e^{i u A(t)} B(t)\right) d t .
$$

Moreover, (12) implies that

$$
\left\|e^{i u A(s)}-e^{i u A\left(s_{0}\right)}\right\|_{1} \leq|u| \int_{s_{0}}^{s}\|B(t)\| d t .
$$


Let $\hat{f}$ be the Fourier transform of $f$, so that, by the functional calculus,

$$
f(A(s))=(2 \pi)^{-1 / 2} \int \hat{f}(u) e^{i u A(s)} d u .
$$

Since $f \in C_{0}^{\infty}, \int|u||\hat{f}(u)| d u<\infty$ and thus (14), (15) imply that $f(A(s))-f\left(A\left(s_{0}\right)\right)$ is trace class, and by (14), (13)

$$
\begin{aligned}
g(s) & =\int i u\left(\int_{s_{0}}^{s} \operatorname{Tr}\left(e^{i u A(t)} B(t)\right) d t\right) \hat{f}(u) d u \\
& =\int_{s_{0}}^{s} \operatorname{Tr}\left(f^{\prime}(A(t)) B(t)\right) d t
\end{aligned}
$$

since it is easy to justify the interchange of integrals, and (15) holds for $f^{\prime}$ and $\widehat{f}^{\prime}(u)=i u \hat{f}(u)$.

For potential applications of spectral averaging to random Schrödinger operators, one wants to allow unbounded $A$ 's and $B$ 's with some kind of relative trace class condition. While one can allow general $B$ 's, we will consider the case $A(s)=$ $A_{0}+s B$. The following is an immediate consequence of the spectral mapping theorem, Theorem 1, and

$$
\frac{d}{d s}\left(A_{0}+s B+1\right)^{-1}=\left(A_{0}+s B+1\right)^{-1} B\left(A_{0}+s B+1\right)^{-1} .
$$

Theorem 3. Let $A_{0} \geq 0$ be a positive self-adjoint operator. Let $b$ be a positive quadratic form so that $\left(A_{0}+1\right)^{1 / 2} b\left(A_{0}+1\right)^{-1 / 2}$ is trace class. Define $A(s)$ by $A(s)=A_{0}+s b$, and $d \mu_{s}(\lambda)$ and $d \eta(\lambda)$ by $(7)$. Then $\left(A\left(s_{0}\right)+1\right)^{-1}-\left(A\left(s_{1}\right)+1\right)^{-1}$ is trace class and (8) holds.

Remark. We used the fact that the Krein spectral shift $\xi_{A, C}$ can be defined (via spectral mapping) if $(C+1)^{-1}-(A+1)^{-1}$ is trace class.

By using modified Fourier transforms based on $e^{i u \lambda}$ with $\operatorname{Im} u=$ constant $>0$, one can prove the following extension:

Theorem 4. Let $A_{0} \geq 0, B \geq 0$ with $B$ a form bounded perturbation of $A_{0}$ with relative bound zero. Define $A(s)=A_{0}+s B$ and suppose that for all $s \in\left[s_{0}, s_{1}\right]$ and $\epsilon>0, B e^{-\epsilon A(s)}$ is trace class and $s \mapsto B e^{-\epsilon A(s)}$ is continuous in the trace norm. Then for any $\epsilon>0, e^{-\epsilon A\left(s_{1}\right)}-e^{-\epsilon A\left(s_{0}\right)}$ is trace class, so we can define a Krein spectral shift $\xi_{A\left(s_{0}\right), A\left(s_{1}\right)}(\lambda)$ in the usual way. Define $d \mu_{s}(\lambda)$ and $d \eta(\lambda)$ by $(7)$ (since $B e^{-\epsilon A(s)}$ is trace class, $\operatorname{Tr}\left(B^{1 / 2} E_{A(s)}(\Delta) B^{1 / 2}\right)$ makes sense for any bounded $\Delta)$. Then (8) holds.

As an application, one can take $A_{0}=-\Delta+V_{0}$ and $B=V_{1}$, where $V_{0}, V_{1}$ are suitable potentials (e.g., both uniformly Kato class and $V_{1} \in \ell_{1}\left(L^{2}\right)$; see [8]).

\section{ACKNOWLEDGMENT}

The author would like to thank M. Ben-Artzi for the hospitality of the Hebrew University, where some of this work was done. 


\section{REFERENCES}

1. M. Birman and M. Solomyak, Remarks on the spectral shift function, J. Soviet Math. 3 (1975), 408-419. MR 47:4031

2. I. Gohberg and M.G. Krein, Introduction to the Theory of Linear Nonself-Adjoint Operators in Hilbert Space, Nauka, Moscow, 1965; English transl., Amer. Math. Soc., Providence, RI, 1969. MR 36:3137; MR 39:7447.

3. V.A. Javrjan, A certain inverse problem for Sturm-Liouville operators, Izv. Akad. Nauk Armjan. SSR Ser. Mat. 6 (1971), 246-251. (Russian) MR 46:723

4. S. Kotani, Lyapunov exponents and spectra for one-dimensional random Schrödinger operators, Contemporary Math., vol. 50, Amer. Math. Soc. Providence, RI, 1984, pp. 277-286. MR 88a: 60116

5. M.G. Krein, On the trace formula in perturbation theory, Matem. Sborn. 33 (1953), 597-626. (Russian) MR 15:720b

6. M.G. Krein, On perturbation determinants and a trace formula for unitary and self-adjoint operators, Dokl. Akad. Nauk SSSR 144 (1962), 268-271; English transl., Soviet Math. Dokl. 3 (1962), 707-710. MR 25:2446

7. M.G. Krein, New investigations in the perturbation theory of self-adjoint operators, First Math. Summer School (Kanev, 1963), Part I, "Naukova Dumka", Kiev, 1964, pp. 103-187; English transl., M. G. Krein, Topics in integral and differential equations and operator theory, Birkhäuser, Basel, 1983, pp. 107-172. MR 32:2119; MR 86m:00014

8. B. Simon, Schrödinger semigroups, Bull. Amer. Math. Soc. 7 (1982), 447-526. MR 86b:81001a

9. B. Simon, Spectral analysis and rank one perturbations and applications, CRM Lecture Notes Vol. 8 (J. Feldman, R. Froese, L. Rosen, eds.), Amer. Math. Soc., Providence, RI, 1995, pp. 109-149. MR 97c:47008

10. F. Wegner, Bounds on the density of states in disordered systems, Z. Phys. B 44 (1981), 9-15. MR 83b: 82060

Division of Physics, Mathematics, and Astronomy, California Institute of Technology, Pasadena, California 91125

E-mail address: bsimon@cco.caltech.edu 